\title{
Fluorescence Intermittency in Self-Assembled InP Quantum Dots
}

\author{
Mitsuru Sugisaki, ${ }^{1, *}$ Hong-Wen Ren, ${ }^{1, \dagger}$ Kenichi Nishi, ${ }^{1,2}$ and Yasuaki Masumoto ${ }^{1,3}$ \\ ${ }^{1}$ Single Quantum Dot Project, ERATO, JST, Tsukuba Research Consortium, 5-9-9 Tokodai, Tsukuba, Ibaraki 300-2635, Japan \\ ${ }^{2}$ System Devices and Fundamental Research, NEC Corporation, 34 Miyukigaoka, Tsukuba, Ibaraki 305-8501, Japan \\ ${ }^{3}$ Institute of Physics, University of Tsukuba, Tsukuba, Ibaraki 305-8571, Japan
}

(Received 31 July 2000)

\begin{abstract}
Fluorescence intermittency in InP self-assembled dots is investigated by means of far field imaging and single dot spectroscopy. Based on our observation that blinking dots are found in the vicinity of scratches and the blinking frequency is drastically enhanced under a near-infrared laser irradiation, we attribute the origin of the fluorescence intermittency to a local electric field due to a carrier trapped at a deep localized center in the $\mathrm{Ga}_{0.5} \mathrm{In}_{0.5} \mathrm{P}$ matrix. The validity of this explanation is confirmed by a thermal activation-type behavior of the switching rate and artificial reproduction of the blinking phenomenon by an external electric field.
\end{abstract}

DOI: 10.1103/PhysRevLett.86.4883

Single quantum dot spectroscopy has revealed a lot of novel information hidden behind the inhomogeneously broadened photoluminescence (PL) band. One of the most interesting findings so far reported in quantum dot (QD) systems is the fluctuation of the PL peak energy and intensity with time, which would be unobservable in macroscopic studies. The former phenomenon, called as the spectral diffusion, is observed in CdSe [1-4] and InAlAs [5] QDs, where the PL peak energies from confined excitons and their LO sidebands fluctuate during the time of measurement. The latter phenomenon is referred to as the fluorescence intermittency or random telegraph signal, where the PL intensity switches between two or more discrete levels as the time goes by [1,5-9]. The spectral diffusion and the fluorescence intermittency are tentatively attributed to photoionization or mobile photoactivated nonradiative recombination centers. However, the detailed mechanism of the fluorescence intermittency is still under discussion, especially the location of the carriers during the blinking process and, more importantly, the origin of such large intensity changes have not been clarified yet.

In this Letter, we report on the origin of the fluorescence intermittency derived from a detailed optical study of self-assembled single InP QDs and show that the blinking is due to a local electric field generated by a carrier trapped by a deep localized center in the $\mathrm{Ga}_{0.5} \mathrm{In}_{0.5} \mathrm{P}$ matrix. The validity of our model is confirmed by the thermal activation-type behavior of the switching rate. In addition, a strong enhancement of the blinking rate was observed when a near-infrared (near-IR) laser is irradiated together with a band-to-band excitation laser, which allowed us to estimate the energy level of the localized center. Notably, we clearly demonstrate how to reproduce all the features of the phenomenon artificially [10].

Self-assembled InP QDs sandwiched between two $\mathrm{Ga}_{0.5} \operatorname{In}_{0.5} \mathrm{P}$ barriers $180 \mathrm{~nm}$ thick each were grown on a Si doped $\left(n^{+}\right)$GaAs (001) substrate using a metal-organic vapor phase deposition system [11]. For the single dot spectroscopic study, we adopted a confocal micropho-
PACS numbers: 71.35.-y, 61.46.+w, 73.21.-b, 78.66.Fd

toluminescence ( $\mu$-PL) system [9]. The samples were set on a cold-finger of a liquid He flow-type cryostat. As the excitation light source, the $488 \mathrm{~nm}$ line of a continuous wave (CW) Ar-ion laser and a CW Ti:sapphire laser were used. The sample PL was collected using a microscope objective lens, and then the images were taken by a thermoelectrically cooled charge-coupled device (CCD) camera. For the $\mu$-PL measurements, the sample PL was analyzed using a $50 \mathrm{~cm}$ single monochromator and then detected by a liquid nitrogen cooled CCD camera.

Figures 1(a) and 1(b) show the $\mu$-PL images consecutively observed over the same area of the sample. Each bright spot corresponds to the PL from a single InP QD. As marked by the dotted circles, it was observed that some QDs exhibit the blinking phenomenon, i.e., the PL intensity switches randomly between a high efficiency state (hereafter referred to as "on") and a very weak state ("off"), while the PL from other QDs is stable. It should be mentioned that, even if a blinking QD is in the off state, the $\mu$-PL is not completely quenched, but weak $\mu$-PL remains.
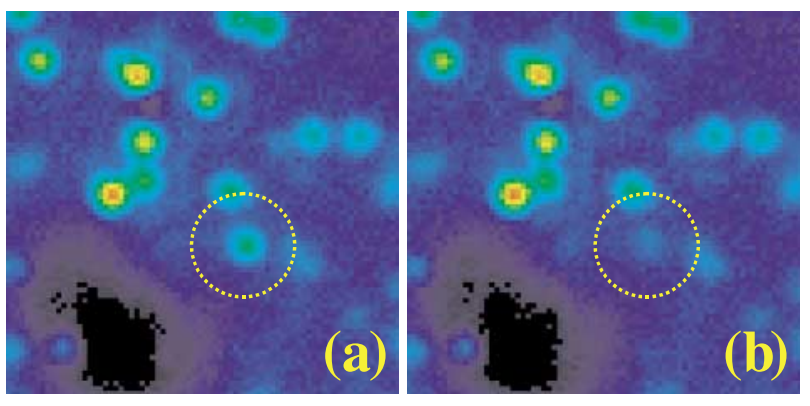

FIG. 1 (color). Microimages of a blinking InP QD in (a) on and (b) off states measured at $4 \mathrm{~K}$ with a $200 \mathrm{msec}$ integration time. The observed area is $15 \mu \mathrm{m} \times 15 \mu \mathrm{m}$. The black region at the bottom left side of each image is a flaw on the sample surface, and thus the PL from the $\mathrm{Ga}_{0.5} \operatorname{In}_{0.5} \mathrm{P}$ matrix is not observed in this region. 


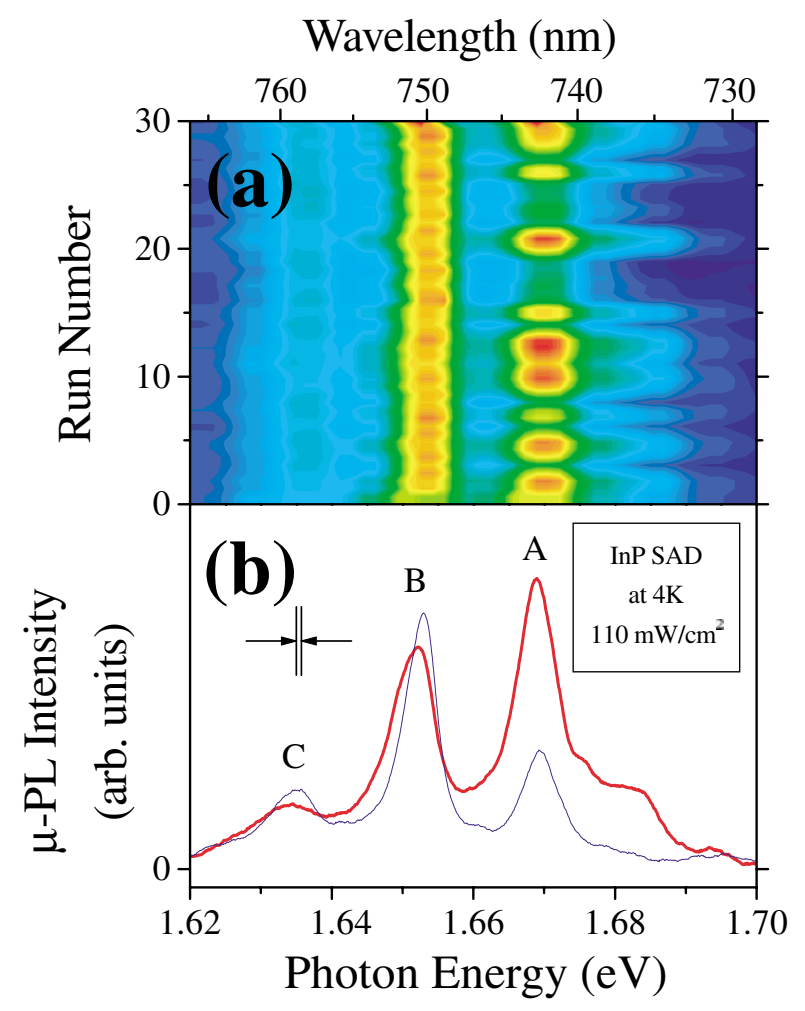

FIG. 2 (color). (a) Contour map made from $\mu$-PL spectra at $4 \mathrm{~K}$ of a blinking InP self-assembled dot (SAD) measured successively 30 times. (b) $\mu$-PL spectra of the on (thick red curve) and off (thin blue curve) states. Peak $A$ having the highest PL peak energy shows the large intensity change between the on and off states. Peaks $B$ and $C$ shift to the higher energy side when the system switches from the on state to the off state, while the intensity changes of these peaks are very small.

Figure 2(a) shows a contour plot of the $\mu$-PL spectra of a single blinking QD successively recorded 30 times with an individual integration time of $200 \mathrm{msec}$. It is found that not only the PL intensity but the $\mu$-PL spectrum also changes with time between the two types of spectra shown by the thick and thin curves in Fig. 2(b). Each of them corresponds to the $\mu$-PL spectrum when the blinking QD is in the on state and the off state, respectively. The PL peak denoted by $A$ shows a large intensity change between the on and off states. On the other hand, the PL bands $B$ and $C$ are almost unchanged in intensity, but the peak energies slightly shift to the higher energy side by a few meV when the state switches from on to off. This result indicates that the large intensity change observed in the $\mu$-PL images is mainly caused by the intensity change of the PL band having the highest PL peak energy. This also explains the weak PL in the off state, viz., some peaks change little when the state switches from on to off so that the $\mu$-PL intensity in the off state, though very weak, is not completely quenched as shown in Fig. 1(b).

In many cases a blinking QD was observed near a minor flaw on the sample surface; for instance, a small flaw is observed at the bottom left side in Fig. 1. It is thus reasonable to assume that there are many defect states near that point. Further, we found that new blinking QDs appeared after the surface of the sample was intentionally scratched by a needle. Therefore the fluorescence intermittency appears to be related to deep defect levels.

A possible mechanism for the switching between the on and off states can be modeled as follows. In the vicinity of some QDs there are localized deep levels due to defects. When a carrier is trapped by such a localized state, a local electric field generated by the trapped carrier is applied to the QD. In this case, the overlap of the wave functions of the confined electrons and holes is decreased. If the local field is much stronger, the confined carriers escape from the QD to the matrix. Therefore the $\mu$-PL intensity eventually becomes weak, and this is observed as the switching from the on state to the off state. The trapped carrier relaxes after a while by recombining with another carrier of opposite sign, mediated by phonons or through a photoreabsorption process. In this case, the local field by the trapped carrier disappears, and the system thus returns to the initial condition. This shows the switching from the off state to the on state [12].

In order to confirm the validity of the proposed model, we performed several additional experiments, which shed light on local trap centers and local electric fields. First, we measured the temperature dependence of the integrated $\mu$-PL intensity as shown in Fig. 3(a). If the switching is due to the trapping process of carriers, the trapping probability should be explained using a thermal activation model. As clearly seen, the switching becomes faster with the increase of the temperature. The average off times measured at various temperatures are summarized in Fig. 3(b). We found that the off period $t_{\mathrm{off}}(T)$ at temperature $T$ can be well reproduced using a thermal activation function,

$$
t_{\mathrm{off}}(T)=\frac{t_{\mathrm{off}}(0)}{1+a \exp \left(-E / k_{\mathrm{B}} T\right)},
$$

(a)

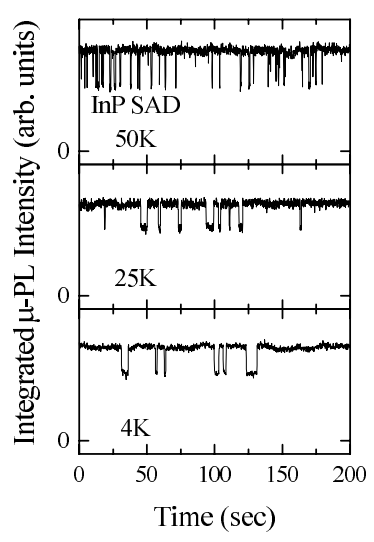

(b)

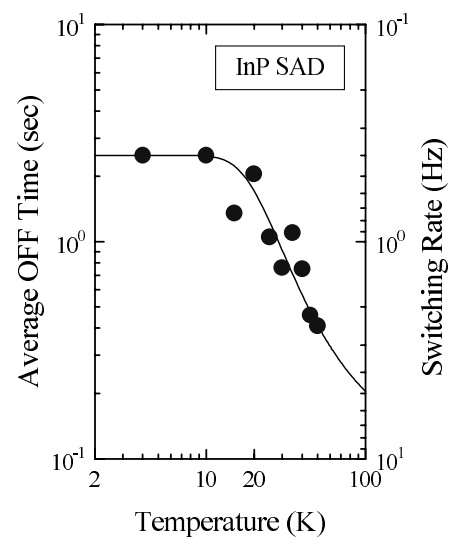

FIG. 3. (a) Time traces of the integrated $\mu$-PL intensity of a blinking QD measured at various temperatures. The switching rate becomes faster as the temperature is increased. (b) Log-log plot of the off duration as a function of temperature, which can be well explained by a thermal activation model written in Eq. (1), with $t_{\text {off }}(0)=2.5 \mathrm{sec}$ and $E=7 \mathrm{meV}$. 
as shown by the solid line in Fig. 3(b), where $a$ is a constant and $E$ and $k_{\mathrm{B}}$ denote the activation energy and the Boltzmann constant, respectively. This result strongly supports the existence of a localized trap center which is responsible for the switching phenomenon.

We then investigated the depth of the localized center. Figure 4(a) shows the integrated $\mu$-PL intensity under weak excitation by an Ar-ion laser. As shown in Fig. 4(b), we observed a drastic enhancement of the on-off switching rate when the QDs were resonantly excited by a near-IR (Ti:sapphire) laser of $1.75 \mathrm{eV}$ in addition to the band-to-band excitation (Ar-ion) laser.

The switching rates which are defined as the reciprocal duration of the on and off periods are plotted in Fig. 4(c) as a function of the excitation energy of the near-IR laser. For comparison, the $\mu$-PL spectrum of the blinking QD is also shown. The broken and dotted lines show the switching rates of the on state and the off state, respectively, measured without the near-IR laser irradiation. When the near-IR laser energy is above $1.5 \mathrm{eV}$, the switching rates of the on and off states become 5 and 3 times faster, respectively, than those excited only by the band-to-band laser [16]. It should be mentioned that the enhancement can be observed even when the near-IR laser energy is below the lowest PL level of the InP QD because the near-IR laser can directly excite a carrier to the defect level in the $\mathrm{Ga}_{0.5} \mathrm{In}_{0.5} \mathrm{P}$ matrix that is deeper than the confined exciton level in the InP QDs. The switching rates were almost independent of the excitation energy of the near-IR laser above $1.5 \mathrm{eV}$. When the near-IR laser energy is lower than $1.5 \mathrm{eV}$, however, the enhancement was not observed, and the switching rate was almost the same as that of Fig. 4(a). Hence we
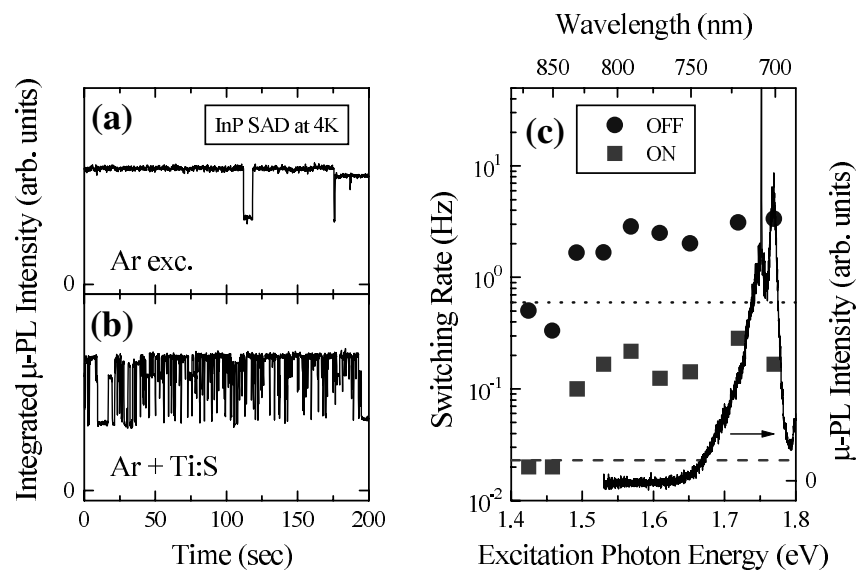

FIG. 4. (a) The integrated $\mu$-PL intensity versus time trace of a blinking QD under band-to-band (Ar-ion laser) excitation. (b) The blinking rate is drastically enhanced when a near-IR (Ti:sapphire) laser beam irradiates the sample simultaneously with a band-to-band excitation laser beam. (c) Plot of the switching rates of the on (squares) and off (circles) states. The broken and dotted curves show the switching rates of the on and off times, respectively, measured without the near-IR laser irradiation. For comparison, the $\mu$-PL spectrum of the blinking QD is also shown. The sharp line observed at $1.75 \mathrm{eV}$ comes from the scattered light of the near-IR laser. conclude that the localized center responsible for blinking has an excitation threshold energy of $1.5 \mathrm{eV}[17,18]$.

Finally we checked the second key point of our model, namely, the local electric field, which is considered to cause a strong change in $\mu$-PL intensity. Figure 5(a) shows the contour map of the $\mu$-PL spectra of a normal single QD showing stable PL measured by applying a positive bias at the top of the sample. In order to perform this experiment, a semitransparent contact was fabricated by evaporating a $20 \mathrm{~nm}$ thick Au layer onto the sample surface. The PL observed at the higher energy side changes more sensitively than those observed at the lower energy side in intensity. As a result, the external electric field causes a fairly large change of the integrated $\mu$-PL intensity as shown in the inset in Fig. 5(b). The thick and thin curves in Fig. 5(b) were measured under the bias of $850 \mathrm{mV}$, which corresponds to a flat band condition $(0 \mathrm{kV} / \mathrm{cm})$, and about $10 \%$ weaker $(750 \mathrm{mV}=-3 \mathrm{kV} / \mathrm{cm})$, respectively [19]. One can see that a small change of the electric field induces a drastic change of the $\mu$-PL spectra. One of the most important points in this study is that the change in intensity and peak energy of the $\mu$-PL spectra in the electric field qualitatively well reproduce the change of $\mu$-PL spectra between

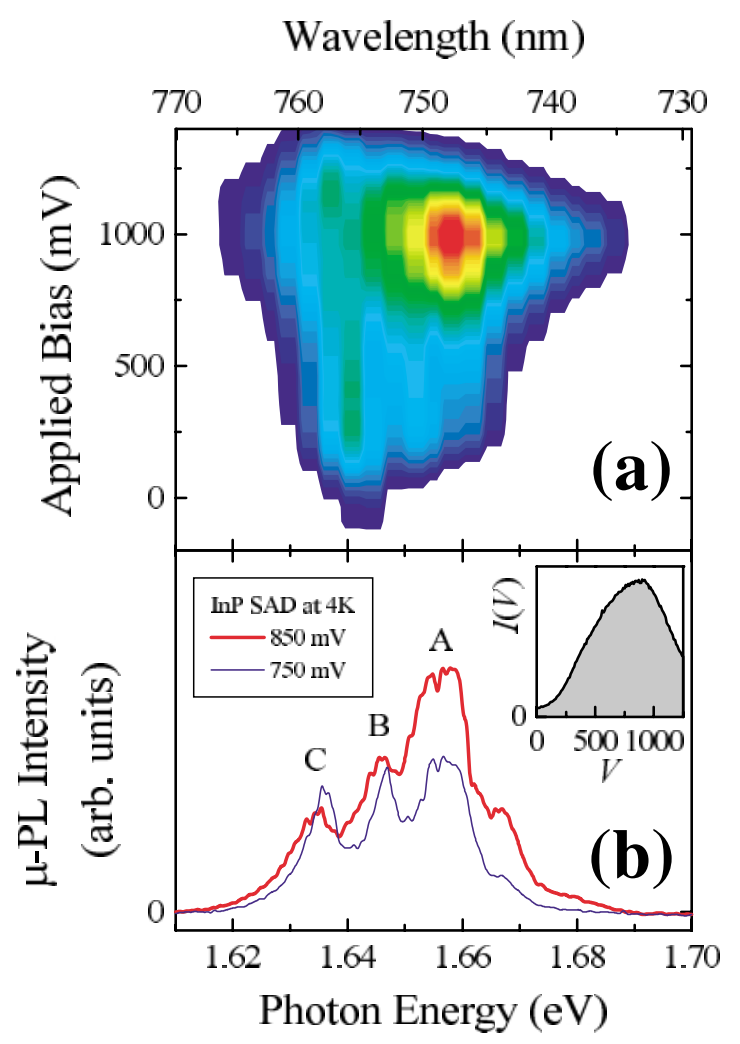

FIG. 5 (color). (a) Contour plot of the $\mu$-PL spectra in an external electric field. The PL intensity of the peak observed at the higher energy side is more sensitive to the field than those observed at the lower energy side. (b) $\mu$-PL spectra measured by applying a bias of $850 \mathrm{mV}$ (the flat band condition, thick red curve) and $750 \mathrm{mV}$ (thin blue curve), which qualitatively reproduce the $\mu$-PL spectra of the on and off states, respectively [compare with Fig. 2(b)]. Inset: Integrated $\mu$-PL intensity versus the applied bias. 
the on and off states of a blinking QD shown in Fig. 2(b). That is, in Fig. 5(b), the PL peak $A$ observed at the higher energy side shows the largest change in intensity, and the PL peaks $B$ and $C$ shift to the higher energy side by a few meV keeping their PL intensities almost unchanged when the bias is changed from 850 to $750 \mathrm{meV}$. This fact is direct evidence supporting our model and brings us to the conclusion that the fluorescence intermittency is due to a local electric field induced by a trapped carrier near a single QD. Furthermore, we can estimate the distance between a blinking QD and a trap center from this result; if one assumes a static electric field in the sample layer and that one localized center can accept only one carrier, the distance is estimated to be $20 \mathrm{~nm}$. This result indicates that the trap center located very close to (but spatially separated by a potential barrier from) the QD plays an important role in the blinking phenomenon.

In summary, we have studied the optical properties of the blinking self-assembled QDs. From the observation of the $\mu$-PL image and the temperature dependence of the switching rate, it has been shown that the blinking phenomenon is due to the trapping and subsequent delocalization processes of photoexcited carriers. Further, we have successfully demonstrated that the intensity changes and peak energy shifts observed in the $\mu$-PL spectra of a blinking QD can be well reproduced artificially by applying an external electric field, which indicates that the fluorescence intermittency is intimately related to a local electric field.

The authors thank Dr. S. V. Nair for many enlightening discussions and a critical reading of the manuscript. Stimulating discussions with Professor I. S. Osad'ko and Dr. J.-S. Lee are also acknowledged.

*Corresponding author.

Email address: mitsuru@ecf.utoronto.ca

Present address: Energenius Centre for Advanced Nanotechnology, University of Toronto, Haultain Building, 170 College Street, Toronto, Ontario, Canada M5S 3E3.

†Present address: Space Vacuum Epitaxy Center, University of Houston, 4800 Calhoun, Houston, TX 77204-5507.

[1] M. Nirmal, B. O. Dabbousi, M. G. Bawendi, J. J. Macklin, J. K. Trautman, T. D. Harris, and L. E. Brus, Nature (London) 383, 802 (1996).

[2] S. A. Empedocles, D. J. Norris, and M. G. Bawendi, Phys. Rev. Lett. 77, 3873 (1996); S. A. Empedocles and M. G. Bawendi, Science 278, 2114 (1997).

[3] V. Türck, S. Rodt, O. Stier, R. Heitz, R. Engelhardt, U. W. Pohl, D. Bimberg, and R. Steingrüber, Phys. Rev. B 61, 9944 (2000).

[4] J. Seufert, R. Weigand, G. Bacher, T. Kümmell, A. Forchel, K. Leonardi, and D. Hommel, Appl. Phys. Lett. 76, 1872 (2000).
[5] H. D. Robinson and B. B. Goldberg, Phys. Rev. B 61, R5086 (2000).

[6] M.-E. Pistol, P. Castrillo, D. Hessman, J. A. Prieto, and L. Samuelson, Phys. Rev. B 59, 10725 (1999).

[7] D. Bertram, M. C. Hanna, and A. J. Nozik, Appl. Phys. Lett. 74, 2666 (1999).

[8] F. Koberling, A. Mews, and T. Basché, Phys. Rev. B 60, 1921 (1999).

[9] M. Sugisaki, H.-W. Ren, S. V. Nair, J.-S. Lee, S. Sugou, T. Okuno, and Y. Masumoto, J. Lumin. 87-89, 40 (2000).

[10] In order to confirm the reproducibility, every phenomenon reported here was examined at least more than three times using different QDs on different days.

[11] H.-W. Ren, M. Sugisaki, J.-S. Lee, S. Sugou, and Y. Masumoto, Jpn. J. Appl. Phys. 38, 507 (1999).

[12] If one assumes that the trapping occurs through the Auger process [13] and if the Auger coefficient is the same order as that reported by Raymond et al. [14], the duration of the on period can be in the same order as our observation. As for the off period, the relaxation time of a single trapped electron observed in several systems is in the same order as the off period in our case [15]. We thus consider that our model is reasonable to explain the duration of the on and off periods.

[13] Al.L. Efros and M. Rosen, Phys. Rev. Lett. 78, 1110 (1997).

[14] S. Raymond, K. Hinzer, S. Fafard, and J. L. Merz, Phys. Rev. B 61, R16331 (2000).

[15] See, for example, X. Jiang, M. A. Dubson, and J. C. Garland, Phys. Rev. B 42, 5427 (1990); H. H. Mueller and M. Schulz, J. Appl. Phys. 83, 1734 (1998); J. H. Scofield, N. Borland, and D. M. Fleetwood, Appl. Phys. Lett. 76, 3248 (2000).

[16] The increase of the temperature by the near-IR laser irradiation is negligible (less than a few $\mathrm{K}$ ) because the redshifts of the $\mu$-PL peak energies of the GaAs substrate and the $\mathrm{Ga}_{0.5} \operatorname{In}_{0.5} \mathrm{P}$ matrix were not observed. Thus, the observed enhancement of the switching rates are not due to the increase of the temperature.

[17] The depth of the deep level that we observe is about $400 \mathrm{meV}$, which is close to the energy due to a Ga-vacancy defect and P-vacancy-related complexes; J. R. Dekker, A. Tukiainen, R. Jaakkola, K. Väkeväinen, J. Lammasniemi, and M. Pessa, Appl. Phys. Lett. 73, 3559 (1998); Z.C. Huang, C. R. Wie, J. A. Varriano, M. W. Koch, and G. W. Wicks, J. Appl. Phys. 77, 1587 (1995).

[18] Further, the anti-Stokes PL due to the deep states with energy $1.5 \mathrm{eV}$ has recently been reported; I. V. Ignatiev, I. E. Kozin, H.-W. Ren, S. Sugou, and Y. Masumoto, Phys. Rev. B 60, R14001 (1999). The value is consistent with that observed in the current study.

[19] A single InP QD shows a $\delta$-function-like $\mu$-PL line under weak excitation [20]. Under the excitation shown in Fig. 5, since the number of the confined excitons is $\sim 10$, the multiple $\mu$-PL lines are observed and the $\mu$-PL band width becomes broad due to the exciton-exciton interaction.

[20] M. Sugisaki, H.-W. Ren, S. V. Nair, K. Nishi, and Y. Masumoto, Solid State Commun. 117, 435 (2001). 